\title{
Solitary upper thoracic vertebral metastasis as an initial presentation of pancreatic cancer: A case report
}

\author{
KALLIOPI ALPANTAKI ${ }^{1}$, CHRISTOS KOUTSERIMPAS ${ }^{2}$, KALLIOPI MILAKI ${ }^{3}$, KONSTANTINOS SPANAKIS ${ }^{4}$, \\ GALATEIA DATSERI $^{5}$, KONSTANTINOS RAPTIS ${ }^{2}$ and GEORGE SAMONIS ${ }^{6}$ \\ ${ }^{1}$ Department of Orthopaedics and Traumatology, 'Venizeleion' General Hospital of Heraklion, Crete 17121; \\ ${ }^{2}$ Department of Orthopaedics and Traumatology, '251' Hellenic Air Force General Hospital of Athens, Athens 11525; \\ ${ }^{3}$ Second Department of Internal Medicine, 'Venizeleion' General Hospital of Heraklion, Crete 17121; ${ }^{4}$ Department of Radiology, \\ University Hospital of Heraklion, Crete 71500; ${ }^{5}$ Department of Pathology, 'Venizeleion' General Hospital of Heraklion, \\ Crete 17121; ${ }^{6}$ Department of Internal Medicine, University Hospital of Heraklion, Crete 71500, Greece
}

Received May 5, 2020; Accepted September 23, 2020

DOI: $10.3892 / \mathrm{mco} .2020 .2199$

\begin{abstract}
The spine represents an unusual site of pancreatic metastatic disease, while the exact incidence of this metastatic lesion is unclear. An extremely rare case of bone painful blastic metastatic lesion at the fourth thoracic vertebra, as a first manifestation of pancreatic cancer, is reported in the current study. A 54-year-old man was complaining of upper thoracic spinal pain that lasted the previous 4 months. A CT scan revealed a solitary, sclerotic, bone lesion of the body of the fourth thoracic (T4) vertebra, indicating a benign lesion. A consequent MRI scan of the thoracic spine confirmed the sclerotic lesion along with additional findings, such as bone marrow edema of the vertebra and a paraspinal and epidural soft tissue mass. Biopsy of the T4 vertebra lesion revealed metastatic lesion of pancreatic origin. A CT scan of the upper abdomen confirmed the diagnosis. This is a rare case, since the only initial symptom of pancreatic cancer was pain of the upper spine, while the first imaging was misleading, indicating a lesion that was benign in nature.
\end{abstract}

\section{Introduction}

Pancreatic cancer usually metastasizes at the liver and the peritoneal cavity. The spine represents an unusual site of pancreatic metastatic disease, while the exact incidence of this metastasis remains unclear (1). These lesions most times are osteolytic. However, lesions of osteoblastic nature, though rare, have also been reported (2). Usually, spine metastases occur in patients with advanced disease, long after the initial diagnosis (2).

Correspondence to: Dr Christos Koutserimpas, Department of Orthopaedics and Traumatology, '251' Hellenic Air Force General Hospital of Athens, Kanellopoulou Avenue 3, Athens 11525, Greece E-mail: chrisku91@hotmail.com

Key words: pancreatic cancer, spinal metastases, spinal lesions
In this report a patient with locally limited pancreatic cancer and initial complain only upper back pain due to a solitary bone blastic metastatic lesion of the fourth thoracic vertebra (T4) is presented.

\section{Case report}

A 54-year-old male presented to the outpatient clinic, complaining for severe upper back pain during the last 4 months. The pain was worse at night, causing severe sleep disturbance, and did not respond to 'over the counter' painkillers. He was stable (blood pressure 120/75 $\mathrm{mmHg}$, heart rate 85 beats/min, $\left.\mathrm{SpO}_{2} 99 \%\right)$ and afebrile $\left(36.6^{\circ} \mathrm{C}\right)$. His medical history included heavy smoking, as well as severe anxiety and panic attacks treated with perphenazine/amitriptyline.

Clinical examination was unremarkable except for tenderness at palpation of the upper thoracic spine. There was no neurological deficit. Laboratory findings included: Hemoglobin=12.7 g/dl, normal white blood cell $(\mathrm{WBC}=5.400)$ and platelet count $(\mathrm{PLT}=210 \mathrm{k} / \mu \mathrm{l})$. Hepatic $(\mathrm{SCOT}=25 \mathrm{IU} / 1$, SGPT=30 IU/1, gGT=76 IU/1 and ALP=95/IU/1) and renal (Cr $1.06 \mathrm{mg} / \mathrm{dl}, \mathrm{UR}=28 \mathrm{mg} / \mathrm{dl}$ ) functions were normal. C-reactive protein $(\mathrm{CRP}=0.43 \mathrm{mg} / \mathrm{dl})$ and prostatic specific antigen $(\mathrm{PSA}=2.96 \mathrm{ng} / \mathrm{ml})$ were within normal range.

CT scan of the thoracic spine revealed a rounded, mostly sclerotic bone lesion, with smooth transitional margins and small inner lucent areas at the T4 vertebral body (Fig. 1). Thus, it was diagnosed by imaging specialists as a benign lesion unrelated to his symptoms.

Three months later, due to persistence of the pain, he underwent a thoracic spine MRI scan, revealing bone marrow edema of part of the T4 vertebral body and presence of paraspinal and epidural soft tissue mass, along with the already known sclerotic lesion (Fig. 2). Since these findings were highly indicative of malignant nature, bone scintigraphy followed which was, surprisingly, normal.

The patient was further investigated with CTs of the thorax and abdomen and a mass infiltrating part of the body and tail of the pancreas indicating pancreatic tumor was revealed. Consequently a $\mathrm{C}$-arm guided biopsy of the $\mathrm{T}-4$ vertebra 


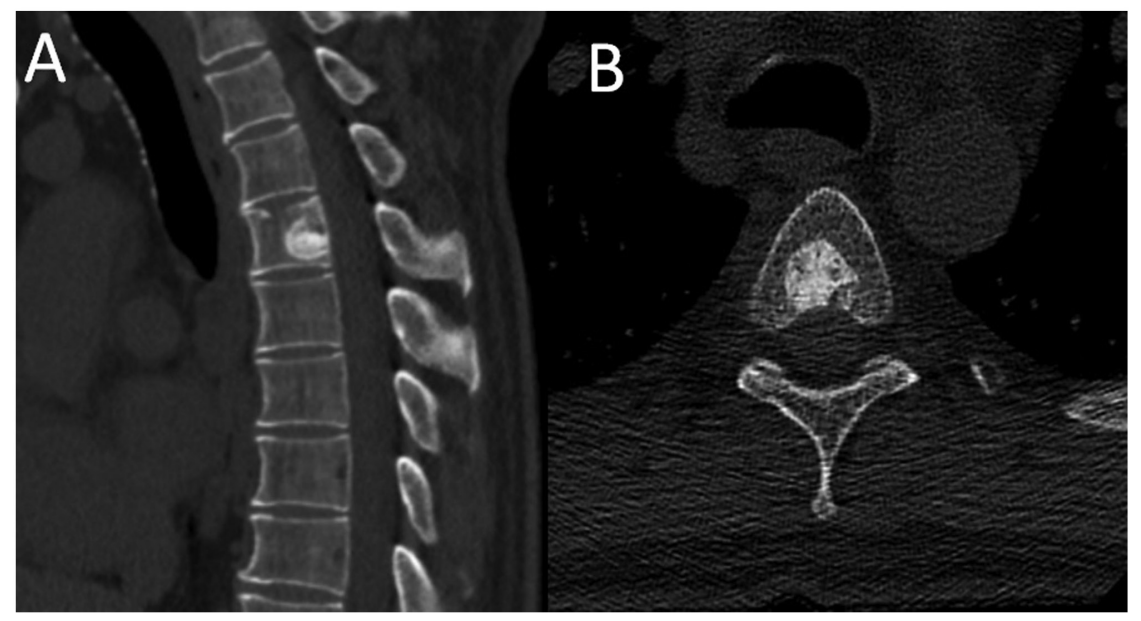

Figure 1. (A) Sagittal reformation CT, (B) Axial CT. Rounded sclerotic lesion in T4 vertebral body with sharp transitional zone, initially considered as benign.

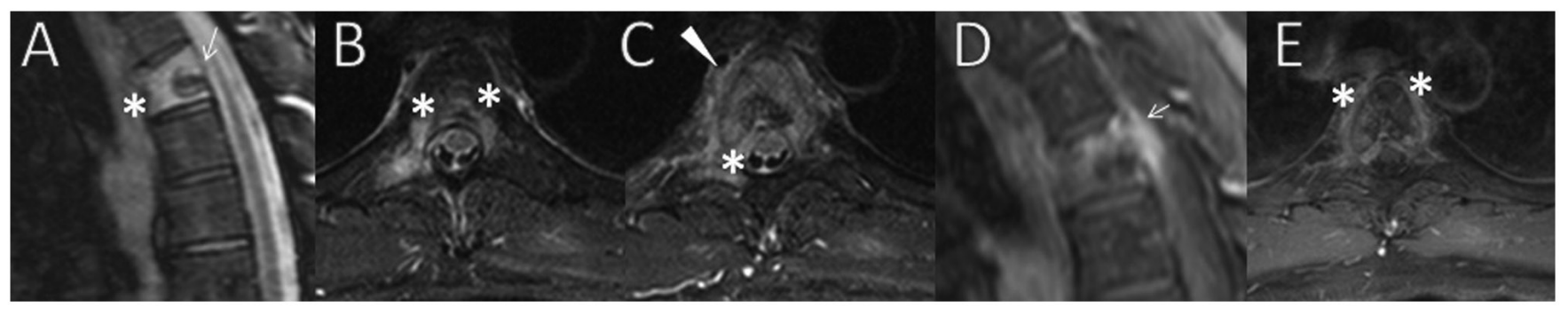

Figure 2. (A) Sagittal STIR MR image shows the sclerotic lesion (arrow) surrounded by bone marrow edema (asterisk). (B and C) Axial STIR MR image. "Extension of the bone marrow edema to the pedicles and right lamina of the vertebra, as long as to the prevertebral soft tissue (arrowhead). (D and E) Axial, sagittal T1-weighted contrast, enhanced MR images illustrates prominent enhancement. "In prevertebral soft tissue and in epidural space (arrows).
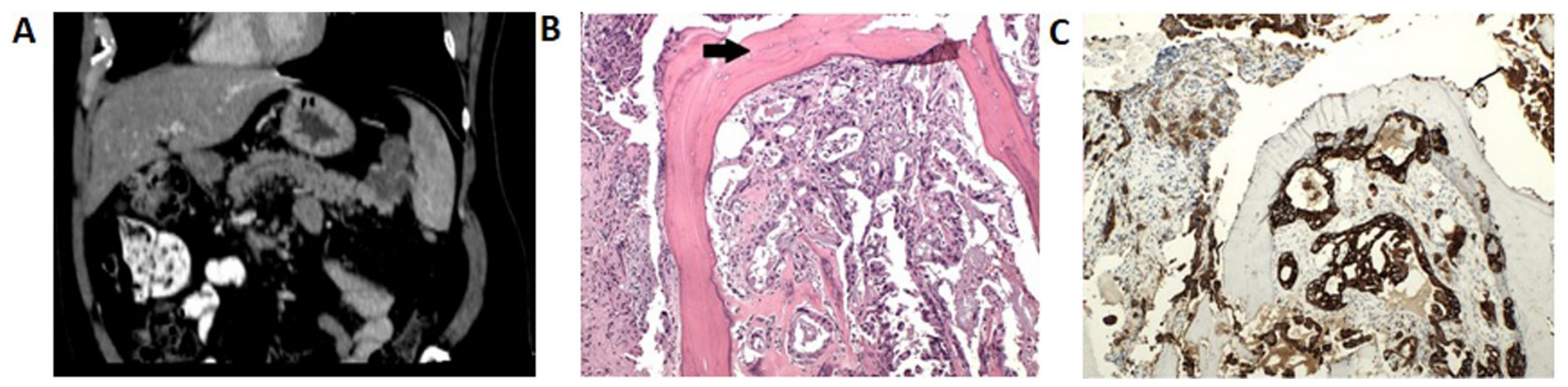

Figure 3. (A) Post contrast upper abdomen CT (coronal view) shows large hypovascular lesion in pancreatic tail, consistent with cancer. (B) Microscopic image from bone (arrow) biopsy, showing extensive infiltration by adenocarcinoma (haemotoxylin and eosin stain, magnification, x100). (C) Microscopic image from bone biopsy, showing strong positivity of the neoplastic cells in cytokeratin 19 immunohistochemical stain (immunohistochemistry; magnification, x100).

revealed histology compatible with pancreatic bone metastasis. In particular, histology demonstrated tumor cells in cavities in-between regenerated bone, consistent with a poor differentiated adenocarcinoma with features indicating pancreatic cancer (Fig. 3).

Hence the diagnosis of pancreatic cancer was confirmed and the patient was referred to the Department of Medical Oncology for antineoplastic treatment. It is of note that, up to that point in time, the patient remained in good condition without weight loss or other usual signs and symptoms related to pancreatic cancer.

\section{Discussion}

Pancreatic cancer, at early stages, is often difficult to be diagnosed due to the absence of specific signs and symptoms; therefore, at the time of diagnosis most patients present with advanced disease (3).

Cardinal symptoms and signs include pain located at the upper abdomen radiating at the back, malaise, weight loss and/or jaundice. Less often patients present with recurrent thrombophlebitis and in rare cases with mental and/or personality disorders (4). Most symptoms are related to the 
location of the primary tumor. Pancreatic body or tail malignancies commonly appear with pain and weight loss, while tumors of the pancreatic head usually present with obstructive jaundice (4). However, the present patient did not have any of these usual symptoms, with the thoracic spinal pain being the only one.

Common metastatic sites include the regional lymph nodes, the liver and less commonly the lungs. Osseous metastases are unusual, while spinal metastatic lesions, as initial presentation, are extremely rare (3). The exact prevalence of bone metastases of this tumor remains unclear. Generally, it is quite rare for the disease to spread outside the abdomen, in the absence of liver disease. Hess et al reported on their tumor registry data from 4,399 patients. In the group of pancreatic adenocarcinoma patients, $85 \%$ of metastases occurred in the liver, $12 \%$ in the lung and $3 \%$ in the skeleton (5). Borad et al, in a retrospective study including 323 patients with pancreatic cancer, reported 7 patients $(2.2 \%)$ with skeletal disease with all of them having spinal involvement (6). Iguchi et al reported 13 patients $(7.3 \%)$ with secondary bone lesions in a retrospective study of 178 patients (7). In the same study, the authors underline that serum levels of the bone resorption marker 1CTP, as well as IL-6 and VEGF were elevated in most cases, while elevation of serum PTHrP levels was found in 3 out of 13 patients. Hence, the authors suggest periodical measurement of serum 1CTP in addition to bone scintigraphy for earlier diagnosis of metastatic bone disease (7).

Bone metastases are mainly lytic. Purely blastic lesions have rarely been reported (2). Ray et al published a case of a solitary osteblastic bone metastasis, in the absence of other systemic disease, occurring 7 years after surgical resection and adjuvant treatment for a locally advanced pancreatic adenocarcinoma (8). The patient had good performance status, with no clinical evidence of recurrence, until an elevation of the CA 19-9 tumor marker initiated a systemic workup. Therefore, an isolated sclerotic lesion in the right sacral ala was identified on imaging and was biopsied, histologically revealing adenocarcinoma, consistent with the primary tumor.

Spinal pain as presenting complaint of metastatic pancreatic cancer is rare, with only few cases in the literature reporting painful vertebral metastatic lesions as first clinical manifestation (9). Pneumaticos et al reported a case of cancer of the body of the pancreas presented with severe back pain due to an osteoblastic lesion to third lumbar vertebra (10). The present patient suffered severe back pain and he had had a CT scan of the upper thoracic spine. A sclerotic lesion had been found, which was misdiagnosed as benign, irrelevant to the patient's symptoms.

Although there are many reliable indicators in the radiological evaluation of a potential osteolytic bone tumor (zone of transition, periosteal reaction, cortical destruction), further investigation should be recommended in cases of atypical osteoblastic lesions, due to lack of similar helpful signs in such lesions (with the exception of a pathognomonic sclerotic bone tumor). Certainly, other clinical symptoms should be sought in the presence of such spinal lesions.

Spinal metastases are rare in pancreatic cancer but they can cause significant morbidity such as excruciating pain and neurological dysfunction, even paralysis, due to neural tissue compression (11). Although the overall prognosis of metastatic pancreatic cancer is very poor, early diagnosis and management of spinal metastases is crucial for the patients' quality of life (10).

In conclusion, pancreatic cancer is not usually included in the differential diagnosis of a bone metastasis of unknown origin. Clinicians should increase their awareness and index of suspicion regarding the types of the disease's presentation and to include pancreatic cancer in the investigation of bone metastases of unknown primary tumor.

\section{Acknowledgements}

Not applicable.

\section{Funding}

No funding was received.

\section{Availability of data and materials}

All data generated or analyzed during this study are included in this published report.

\section{Authors' contributions}

$\mathrm{KA}, \mathrm{CK}$, GS and KR made substantial contribution to the conception and design of the current study. KM, KS, GD acquired and analyzed the data. KA, GS and CK drafted the manuscript. GS, GD, KR, KS critically revised the manuscript. All authors read and approved the final manuscript.

\section{Ethics approval and consent to participate}

Not applicable.

\section{Patient consent for publication}

Informed consent for publication was received from the patient.

\section{Competing interests}

The authors declare that they have no competing interests.

\section{References}

1. Puri A, Chang J, Dragovich T, Lucente P and Kundranda MN: Skeletal metastases in advanced pancreatic ductal adenocarcinoma (PDAC): A retrospective analysis. J Clin Oncol 36: 245, 2018

2. Joffe $\mathrm{N}$ and Antonioli DA: Osteoblastic bone metastases secondary to adenocarcinoma of the pancreas. Clin Radiol 29: 41-46, 1978.

3. Lin CT, Tang CT, Liu MY and Ma HI: Unusual osteoblastic metastases in the spine secondary to adenocarcinoma of the pancreas. Acta Chir Belg 111: 44-45, 2011.

4. Bakkevold KE, Arnesjø B and Kambestad B: Carcinoma of the pancreas and papilla of Vater: Presenting symptoms, signs, and diagnosis related to stage and tumour site. A prospective multicentre trial in 472 patients. Norwegian pancreatic cancer trial. Scand J Gastroenterol 27: 317-325, 1992.

5. Hess KR, Varadhachary GR, Taylor SH, Wei W, Raber MN, Lenzi R and Abbruzzese JL: Metastatic patterns in adenocarcinoma. Cancer 106: 1624-1633, 2006. 
6. Borad MJ, Saadati H, Lakshmipathy A, Campbell E, Hopper P, Jameson G, Von Hoff DD and Saif MW: Skeletal metastases in pancreatic cancer: A retrospective study and review of the literature. Yale J Biol Med 82: 1-6, 2009

7. Iguchi H, Yasuda M, Matsuo T, Sumii T and Funakoshi A: Clinical features and management of pancreatic cancer with bone metastases. Nihon Shokakibyo Gakkai Zasshi 101: 872-878, 2004 (In Japanese)

8. Ray AE, Faltings L, Machnick S, Goenka A, Opher E, Steinberg J, Ratzon F and Novoselac AV: Bone metastasis as the only site of disease in a patient 7 years post treatment for a locally advanced pancreatic adenocarcinoma. J Pancreas 19: 296-302, 2018.

9. Rosenberg E and Buchtel L: Cervical spine pain as a presenting complaint in metastatic pancreatic cancer: A case report. Postgrad Med 128: 331-333, 2016.
10. Pneumaticos SG, Savidou C, Korres DS and Chatziioannou SN: Pancreatic cancer's initial presentation: Back pain due to osteoblastic bone metastasis. Eur J Cancer Care (Engl) 19: 137-140, 2010.

11. Rades D, Huttenlocher S, Schild SE and Bartscht T: Metastatic spinal cord compression from pancreatic cancer. Anticancer Res 34: 3727-3730, 2014.

c) (7) $\Theta$ This work is licensed under a Creative Commons Attribution-NonCommercial-NoDerivatives 4.0 International (CC BY-NC-ND 4.0) License. 